- A useful reminder of a rare occurrence.

- The diagnosis relies on radiology and clinical findings alone.

- Careful follow-up of the patient is required.

\title{
Florid cemento-osseous dysplasia in a young Indian woman. A case report
}

\author{
W. Jerjes, ${ }^{1}$ B. Banu, ${ }^{2}$ B. Swinson ${ }^{3}$ and C. Hopper ${ }^{4}$
}

We present a rare case of florid cemento-osseous dysplasia (FCOD) occurring in a 27-year-old Indian woman. She presented with three lesions, two of them symmetrical, at the posterior region of the mandible. Another lesion appeared nine months later, symmetrical to the existing third lesion. This confirmed the diagnosis of florid cemento-osseous dysplasia as being symmetrical asymptomatic bone lesions occurring in the jaw. The diagnosis was made radiographically depending on a series of radiographs, as biopsy is contraindicated. No treatment was required as the lesions were asymptomatic, causing no problem to the patient. The patient continues to be reviewed annually. The case is rare in its unusual combination of the disease itself (FCOD), age (27 years) and race (Indian). Clinical and radiological features are presented.

\section{CASE REPORT}

A 27-year-old Indian woman was referred by her general dental practitioner to the department of maxillofacial surgery at the Eastman Dental Hospital for further management of incidental radiographic findings of three separate radiolucencies in the periapical regions of the lower right first molar and lower left first molar and premolar teeth. On questioning, the lesions were asymptomatic and no relevant medical, family or social history was elicited.

On clinical examination there was no evidence of dental decay or periodontal

\footnotetext{
${ }^{1 *}$ Clinical Assistant, Department of Oral and Maxillofacia Surgery, Eastman Dental Institute for Oral Health Care Sciences, 256 Gray's Inn Road, London WC1X 8LD;

${ }^{2}$ Specialist in Surgical Dentistry, Department of Oral and Maxillofacial Surgery, Eastman Dental Institute for Oral Health Care Sciences; ${ }^{3}$ Specialist Registrar in Oral and Maxillofacial Surgery, University College London

Hospitals; ${ }^{4}$ Consultant Oral and Maxillofacial Surgeon, Senior lecturer, Head of Oral and Maxillofacial Surgery Department, Eastman Dental Institute for Oral Health Care Sciences.

${ }^{*}$ Correspondence to: Dr Waseem Jerjes

Email:waseem_wk1@yahoo.co.uk
}

\section{Refereed Paper}

Received 09.02.04; Accepted 15.09.04

doi: 10.1038/sj.bdj.4812251

๑ British Dental Journal 2005; 198: 477-478 disease. The teeth were vital in their response to ethyl chloride with only slight tenderness due to minor expansion of the buccal plate in the lower left first molar region being noted. The teeth were not found to be tender to percussion.

Radiological examinations revealed three radiolucent lesions, two of which were symmetrical at presentation. These lesions appeared to have well-defined margins and measured $1.5 \mathrm{~cm}$ in diameter. Opinions from our radiologist colleagues confirmed the provisional diagnosis as cemental dysplasia that could be either periapical or florid (Fig. 1).

Six months later the patient attended a joint endodontic/surgery review.The lesions were of similar size radiographically, with some elements of maturation evident. No active treatment was initiated at this time but annual follow up was proposed for the patient.

Twenty months following the initial presentation, a further follow up showed no change in the clinical picture but radiography (Fig. 2) revealed a $1.5 \mathrm{~cm}$ in diameter radiolucent lesion in the periapical region of the lower right first premolar tooth. This proved to be entirely symmetrical with the lesion on the contralateral first premolar. This new lesion confirmed the diagnosis of florid cemento-osseous dysplasia. ${ }^{1-4}$

Continued follow-up has shown no infiltration of the periodontal ligaments, variable degrees of maturation of the lesions and only minor expansion in the buccal plate of the lower left first molar tooth.

\section{DISCUSSION}

Cemento-osseous dysplasias are a group of disorders known to originate from periodontal ligament tissues and involve, essentially, the same pathological process. They are usually classified, depending on their extent and radiographic appearances, into three main groups: periapical (surrounds the periapical region of teeth and are bilateral), florid (sclerotic symmetrical masses) and focal (single lesion) cemental dysplasias.

The term florid cemento-osseous dysplasia (FCOD) has been proposed in the second edition ${ }^{5}$ of the World Health Organisation's (WHO) 'International histological classification of odontogenic tumours' to replace the first edition's 'gigantiform cementoma' ${ }^{6}$ The disorder is strictly localised to the tooth-bearing areas and not associated with any other skeletal 


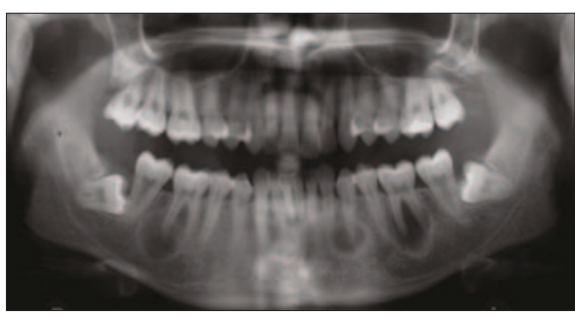

Fig. 1 Diagnosis confirmed as cemental dysplasia

disease. The affected area undergoes changes from normal vascular bone into an avascular cementum-like lesion. The lesion is usually benign and requires no treatment unless cosmetically embarassing; however, treatment of a secondary infection of this lesion can be difficult and complicated. ${ }^{7,8}$ These lesions appear as radio-opaque, irregular or lobulated masses without radiolucent borders interspersed with ill defined radiolucent/radio-opaque areas.

In the past these calcifications have been interpreted as chronic diffuse sclerosing osteomyelitis; Schneider and Mesa ${ }^{9}$ reported two cases of each condition as they tried to demonstrate any differences between them. Chronic diffuse sclerosing osteomyelitis is a primary inflammatory condition of the mandible presenting with cyclic episodes of unilateral pain and swelling and shows a single area of diffuse sclerosis containing small, ill-defined osteolytic areas. These do not usually occur in florid cemento-osseous dysplasia while histologically they are the same entity. ${ }^{10,11}$

Florid cemento-osseous dysplasia may have similarities with jaw bone changes in familial adenomatosis coli (Gardner's syndrome), ${ }^{12}$ but florid cemento-osseous dysplasia has no other skeletal changes or skin tumours or even the dental anomalies that are seen in this syndrome.

Another radiographic differential diagnosis is Paget's disease, ${ }^{13}$ but the dysplastic lesions in Paget's are polystotic and the disease also shows biochemical serum changes.

MacDonald and Jankowski published a systematic review (SR) on FCOD, ${ }^{14}$ with particular regard to comparison between oriental and non-oriental population. He reviewed a series of 156 female cases that were observed in 17 series of patients reported in SR-identified reports. 59.6\% (93 patients) were black, 37.2\% (58 patients)

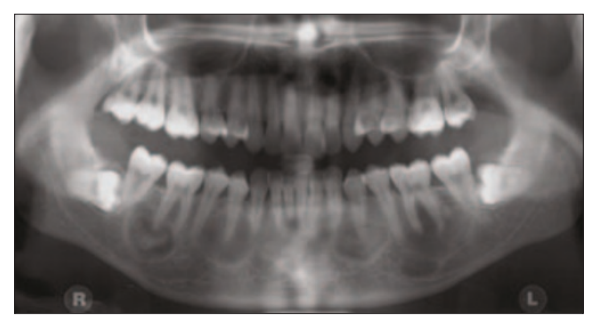

Fig. 2 Symmetrical jaw lesions confirmed the diagnosis as FCOD

were oriental and 3.2\% (5 patients) were Caucasians and Indians. Only three Indian patients were identified from the whole series that combined most of the cases reported around the world; this makes the occurrence of FCOD (that is considered relatively a rare disease) in the Indian race less than $2 \%$. The same systematic review study found that the mean age for those patients was 47-49 years old.

This case was monitored with a series of radiographs (orthopantomograms, periapicals and anterior and posterior occlusals). A biopsy is not required to confirm the diagnosis as this is usually established radiographically. Indeed biopsy increases the risk of infection or fracture of the jaw and it is not normally justified to surgically remove these lesions, as this often requires extensive surgery.

The current methods of treating FCOD are not completely clear cut. If it is a benign condition and no complications exist it may be left untreated, but at the same time the patient should undergo clinical and radiographic follow-up for several years to ensure there is no change in the behaviour of the lesion.

Waldron et al. ${ }^{15}$ observed patients who presented with poor socket healing and even sequestrum formation following extraction of teeth that were closely associated with large cemental masses, and some other groups also recommend that every effort should be made to avoid extraction in patients with FCOD. ${ }^{7,8,15}$

The onset of symptoms is usually a sign that the lesion may be inflamed (chronic osteomyelitis); in this case administration of antibiotics is indicated. It is been proved that the risk of osteomyelitis is increased because of the impaired blood circulation in the affected area of bone.

Under these circumstances, surgery becomes necessary. The extent of intervention may be significant as incomplete resection of these lesions may allow infection to persist and possibly involve other areas of cemento-osseous dysplasia.

\section{CONCLUSION}

Normally, pathology in the jaws requires a diagnosis based on clinical findings, radiographic features and histology. However, florid cemento-osseous dysplasia is a condition in which the diagnosis relies on radiology and clinical findings alone. As reassuring as a biopsy might be, in this situation it may precipitate infection that is difficult to control without extensive surgical intervention.

1. Waldron C A. Fibro-osseous lesions of the jaws. J Oral Maxillofac Surg 1993; 51(8): 828-835.

2. Damm D D, Fantasia J E. Multifocal mixed radiolucencies. Florid cemento-osseous dysplasia. Gen Dent 2001; 49(5): 461, 538.

3. Baughman R, Cook L. Diagnostic quiz \#33. Case no. 1. Florid cemento-osseous dysplasia. Todays FDA 1999; 11(6): 5C, 7C.

4. Slootweg PJ. Maxillofacial fibro-osseous lesions: classification and differential diagnosis. Semin Diagn Pathol 1996; 13(2): 104-112.

5. Kramer I R H, Pindborg J J, Shear M. Histological typing of odontogenic tumours. World Health Organization, International histological classification of tumours (2nd edn). Berlin, Germany: Springer-Verlag, 1992

6. Pindborg J J, Kramer I R H, Torloni H. Histological typing of odontogenic tumours, jaw cysts and allied lesions. International histological classification of tumours. pp 32-34. Geneva: World Health Organization, 1971.

7. Waldron C A. Fibro-osseous lesions of the jaws. J Oral Maxillofac Surg 1985; 43: 249-262.

8. Bencharit S, Schardt-Sacco D, Zuniga J R, Minsley G E. Surgical and prosthodontic rehabilitation for a patient with aggressive florid cemento-osseous dysplasia: a clinical report. J Prosthet Dent 2003; 90(3): 220-224.

9. Schneider LC, Mesa M L. Differences between florid osseous dysplasia and chronic diffuse sclerosing osteomyelitis. Oral Surg Oral Med Pathol 1990; 70: 308-312.

10. Slootweg P J, Muller H. Differential diagnosis of fibro-osseous jaw lesions. A histological investigation on 30 cases. J Craniomaxillofac Surg 1990; 18(5): 210-4.

11. El-Mofty S. Chronic diffuse sclerosing osteomyelitis. Oral Surg Oral Med Oral Pathol 1973; 36: 898-904.

12. Wolf J, Jarvinen H J, Hietanen J. Gardner's dentomaxillary stigmas in patients with familial adenomatosis coli. Br J Oral Maxillofac Surg 1986; 24(6): 410-416.

13. Winer H J, Goepp R A, Olson R E. Gigantiform cementoma resembling Paget's disease: report of case. J Oral Surg 1972; 30(7): 517-519.

14. MacDonald-Jankowski D S. Florid cemento-osseous dysplasia: a systematic review. Dentomaxillofac Radio/ 2003; 32(3): 141-149.

15. Waldron C A, Giansanti J S, Browand B C. Sclerotic cemental masses of the jaws (so called chronic diffuse sclerosing osteomyelitis, sclerosing osteitis, multiple enostosis and gigantiform cementoma). Oral Surg Oral Med Oral Pathol 1975; 39: 590-604. 